\title{
Metabolic engineering of methylotrophic Pichia pastoris for the production of $\beta$-alanine
}

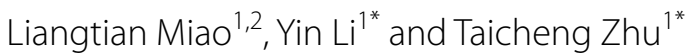

\begin{abstract}
$\beta$-Alanine (3-aminopropionic acid) is the only naturally occurring $\beta$-amino acid and an important precursor for the synthesis of a variety of nitrogen-containing chemicals. Fermentative production of $\beta$-alanine from renewable feedstocks such as glucose has attracted significant interest in recent years. Methanol has become an emerging and promising renewable feedstock for biomanufacturing as an alternative to glucose. In this work, we demonstrated the feasibility of $\beta$-alanine production from methanol using Pichia pastoris (Komagataella phaffii) as a methylotrophic cell factory. L-Aspartate-a-decarboxylases (ADCs) from different sources were screened and expressed in P. pastoris, followed by the optimization of aspartate decarboxylation by increasing the ADC copy number and C4 precursor supply via the overexpression of aspartate dehydrogenase. The production potential of the best strain was further evaluated in a 1-L fermenter, and a $\beta$-alanine titer of $5.6 \mathrm{~g} / \mathrm{L}$ was obtained. To our best knowledge, this is the highest metabolite production titer ever reached in P. pastoris using methanol as the substrate.
\end{abstract}

Keywords: Pichia pastoris (Komagataella phaffii), $\beta$-Alanine (3-aminopropionic acid), Methanol, Aspartate decarboxylation, Aspartate dehydrogenase

\section{Introduction}

$\beta$-Alanine (3-aminopropionic acid) is a naturally occurring $\beta$-amino acid that serves as a precursor for the biosynthesis of a variety of nitrogen-containing chemicals, such as D-pantothenic acid (vitamin B5) (Tigu et al. 2018), coenzyme A (CoA) (Tomita et al. 2014), carnosine (Harris et al. 2006; Sale et al. 2010), and polyalanine (nylon-3) (Steunenberg et al. 2013). $\beta$-Alanine can be chemically synthesized via the ammonification of acrylonitrile (Carlson 1943), hydrolyzation of $\beta$-aminopropionitrile in the presence of barium hydroxide (Ford 1945), or the reaction of acrylic acid with ammonium carbonate and $\mathrm{CO}_{2}$ (Ohara et al. 2011). However, these chemical synthesis routes are not sustainable due to the use of non-renewable substrates and

\footnotetext{
*Correspondence: yli@im.ac.cn; zhutc@im.ac.cn

${ }^{1}$ CAS Key Laboratory of Microbial Physiological and Metabolic

Engineering, State Key Laboratory of Microbial Resources, Institute of Microbiology, Chinese Academy of Sciences, Beijing 100101, People's Republic of China

Full list of author information is available at the end of the article
}

harsh reaction conditions, and biological processes for $\beta$-alanine synthesis have gained increasing interest.

Biological production of $\beta$-alanine is achieved through biotransformation or fermentation. In the biotransformation route, $\beta$-alanine can be directly synthesized from $\mathrm{L}$-aspartic acid via decarboxylation by L-aspartate- $\alpha$-decarboxylase (ADC, EC: 4.1.1.11) ( $\mathrm{Li}$ et al. 2018; Pei et al. 2017; Shen et al. 2014), or indirectly synthesized from fumaric acid as a cheaper substrate, via the consecutive action of aspartate ammonia-lyase (AspA, EC 4.3.1.1) and ADC (Qian et al. 2018; Wang et al. 2020). Although a very high $\beta$-alanine titer (up to $200 \mathrm{~g} / \mathrm{L}$ ) has been reached with whole-cell transformation, the process is not entirely sustainable due to the use of expensive precursors or petrochemicals (i.e., L-aspartic acid or fumaric acid) as substrates. Consequently, the fermentation route has been pursued to synthesize $\beta$-alanine from renewable feedstocks. The fermentative production of $\beta$-alanine from glucose has been described in several reports on the metabolic engineering of Escherichia coli, and promising 
$\beta$-alanine titers ranging from 32.3 to $43.12 \mathrm{~g} / \mathrm{L}$ were obtained in these studies (Piao et al. 2019; Song et al. 2015; Zou et al. 2020).

Although glucose is the most widely used raw material for the production of biochemicals, the exploration of alternative feedstocks has remained a central task in the research on biomanufacturing (Liu et al. 2021). Methanol is considered as one of the most promising feedstocks due to its unique advantages such as providing highly reduced carbon, not competing with food sources, and potentially sustainable production in the future (Zhu et al. 2020). Consequently, there is an emerging trend of utilizing methanol as an alternative feedstock for chemical production using natural or synthetic methylotrophs (Guo et al. 2021; Jin et al. 2021; Tuyishime et al. 2018; Whitaker et al. 2017; Zhu et al. 2016).

In this work, we aimed to achieve the production of $\beta$-alanine from methanol using Pichia pastoris (Komagataella phaffi) as the methylotrophic cell chassis (Fig. 1). ADCs from different sources were screened and expressed in P. pastoris, followed by the optimization of aspartate decarboxylation and $\mathrm{C} 4$ precursor supply, and the best strain reached a $\beta$-alanine titer of $5.6 \mathrm{~g} / \mathrm{L}$. To our best knowledge, this is the first report demonstrating the feasibility of using $P$. pastoris as the chassis for the production of amino acids from methanol.

\section{Materials and methods}

Strains, media, and growth conditions

The primers, strains and plasmids used in this study are listed in Table 1 and Table 2. For E. coli strain construction, the cells were cultured aerobically at $37{ }^{\circ} \mathrm{C}$ in Luria-Bertani (LB) broth $(10 \mathrm{~g} / \mathrm{L}$ tryptone, $5 \mathrm{~g} / \mathrm{L}$ yeast extract and $10 \mathrm{~g} / \mathrm{L} \mathrm{NaCl}$ ). For the construction of $P$. pastoris strains, YPD $(20 \mathrm{~g} / \mathrm{L}$ peptone, $10 \mathrm{~g} / \mathrm{L}$ yeast extract and $20 \mathrm{~g} / \mathrm{L}$ glucose $)$ or MD (20 g/L glucose, $13.4 \mathrm{~g} / \mathrm{L}$ yeast nitrogen base and $0.4 \mathrm{mg} / \mathrm{L}$ biotin) medium was used, and the cells were cultured aerobically at $30{ }^{\circ} \mathrm{C}$. Kanamycin $(50 \mathrm{mg} / \mathrm{L}), \mathrm{G} 418(200 \mathrm{mg} / \mathrm{L})$, Zeocin $(50 \mathrm{mg} / \mathrm{L})$ or hygromycin $(50 \mathrm{mg} / \mathrm{L})$ were added where appropriate.

\section{Construction of recombinant plasmids}

The P. pastoris HIS4 gene was amplified by PCR from pAO $\alpha$ vector (Zhu et al. 2011) with the primer pair His4BamHI-F/His4-BamHI-R, which was then digested with BamHI and ligated into the BamHI site of pPICZA to generate the expression vector pMPICZHis. The zeocin resistance marker of pMPICZHis was replaced with the kanamycin or hygromycin resistance gene using a

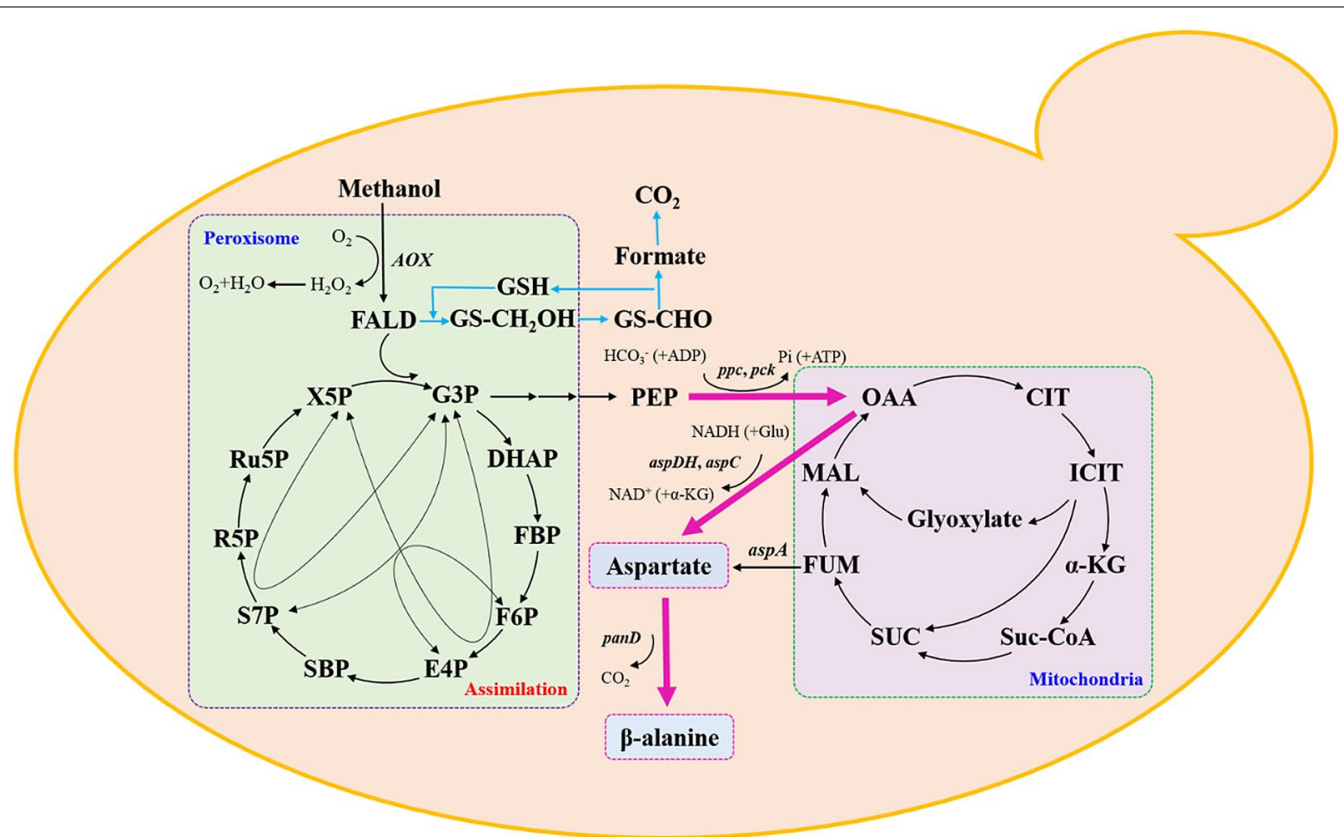

Fig. 1 The biosynthesis pathway designed in P. pastoris for the production of $\beta$-alanine from methanol. AOX: gene encoding alcohol oxidase; $p p c$ : gene encoding PEP carboxylase; $p c k$ : gene encoding PEP carboxykinase; aspDH: gene encoding aspartate dehydrogenase; aspC: gene encoding aspartate aminotransferase; aspA: gene encoding aspartate ammonia-lyase; panD: gene encoding aspartate-a-decarboxylases; FALD: formaldehyde; X5P: xylulose-5-phosphate; G3P: glyceraldehyde-3-phosphate; DHAP: dihydroxyacetone phosphate; FBP: fructose-1,6-bisphosphate; F6P: fructose-6-phosphate; E4P: erythrose-4-phosphate; SBP: sedoheptulose-1,7-bisphosphate; S7P: sedoheptulose-7-phosphate; R5P: ribose-5-phosphate; Ru5P: ribulose-5-phosphate; PEP: phosphoenolpyruvate; Glu: glutamate; OAA: oxaloacetate; CIT: Citrate; ICIT: isocitrate; a-KG: a-ketoglutarate; Suc-CoA: succinyl-CoA; SUC: succinate; FUM: fumarate; MAL: malate 
Table 1 Primers used in this study

\begin{tabular}{|c|c|}
\hline Primers name & Sequences $\left(5^{\prime} \rightarrow 3^{\prime}\right)$ \\
\hline $5^{\prime} \mathrm{AOX} 1$ & GACTGGTTCCAATTGACAAGC \\
\hline $3^{\prime} \mathrm{AOX} 1$ & GCAAATGGCATTCTGACATCC \\
\hline His4-BamHI-F & GCATGGATCCATGACATTTCCCTTGCTAC \\
\hline His4-BamHI-R & GCATGGATCCGTTAAATAAGTCCCAGTTTCTC \\
\hline pMPICZA-3881-F & GGTTTAGTTCCTCACCTTG \\
\hline pMPICZA-4256-R & TCCCCCTTTTCCTTTGTC \\
\hline Kan-pMPICZA-F & ACAAGGTGAGGAACTAAACCATGAGCCATATTCAACGG \\
\hline Kan-pMPICZA-R & TCGACAAAGGAAAAGGGGGATTAGAAAAACTCATCGAGC \\
\hline Hyg-pMPICZA-F & ACAAGGTGAGGAACTAAACCATGAAAAAGCCTGAACTCAC \\
\hline Hyg-pMPICZA-R & TCGACAAAGGAAAAGGGGGACTATTCCTTTGCCCTCGG \\
\hline pTEF1-F & GCAATCTAATCTAAGGGGCGGTGT \\
\hline CYC1TT-R & TTCGAGCGTCCCAAAACCTTCTCAAGC \\
\hline ADCBs-Xhol-F & GCATCTCGAGATGGATGTATCGAACAATGATGAGC \\
\hline ADCBs-Notl-R & GCATGCGGCCGCCTACAAAATTGTACGGGCTG \\
\hline Oan-Xhol-F & GCATCTCGAGATGTCCGTATCTGAAACC \\
\hline Oan-Notl-R & GCATGCGGCCGCTCAGATAACGGTAGTTGCTACGC \\
\hline Spe-Xhol-F & GCATCTCGAGATGAAAAAAATCATGATGATCGG \\
\hline Spe-Notl-R & GCATGCGGCCGCTTATGCGATAAAGCCACCGTC \\
\hline AAT1-BstBI(gb)-F & ATCAAAAAACAACTAATTATTCGAAATGTCGTTTTCAACGCAGAA \\
\hline AAT1-Notl(gb)-R & AGTTTTTGTTCTAGAAAGCTGGCGGCCGCCTATACACGCACAACCTGAT \\
\hline AAT2-BstBI(gb)-F & ATCAAAAAACAACTAATTATTCGAAATGTCGTTTTCAACGCAGAA \\
\hline AAT2-Notl(gb)-R & AGTTTTTGTTCTAGAAAGCTGGCGGCCGCCTATACACGCACAACCTGAT \\
\hline AsPCK-BstBl(gb)-F & ATCAAAAAACAACTAATTATTCGAAATGACTGACTTAAACAAACTCG \\
\hline AsPCK-Notl(gb)-R & AGTTTTTGTTCTAGAAAGCTGGCGGCCGCTTATGCTTTTTGGACCGGCGCCAAC \\
\hline$P P C-B s t B I(g b)-F$ & ATCAAAAAACAACTAATTATTCGAAATGAACGAACAATATTCCGC \\
\hline PPC-Notl(gb)-R & AGTTTTTGTTCTAGAAAGCTGGCGGCCGCTTAGCCGGTATTACGCATAC \\
\hline PCK-BstBI(gb)-F & ATCAAAAAACAACTAATTATTCGAAATGCGCGTTAACAATGGTTTG \\
\hline PCK-Notl(gb)-R & AGTTTTTGTTCTAGAAAGCTGGCGGCCGCTTACAGTTTCGGACCAGCCGC \\
\hline
\end{tabular}

Gibson assembly strategy to yield the expression vectors pMPICKmHis and $\mathrm{pMPIC} 2 \mathrm{H}$, respectively. The panD genes encoding ADCs from Bacillus paralicheniformis ATCC 9945a (GenBank: AGN36811.1), Bacillus subtilis (NCBI Reference Sequence: WP_161619406.1), Streptomyces griseorubiginosus (NCBI Reference Sequence: WP_120050174.1), and Corynebacterium glutamicum (NCBI Reference Sequence: WP_003857183.1), the $\operatorname{aspDH}$ genes encoding aspartate dehydrogenase (AspDH) from Ochrobactrum anthropi ATCC 4918 (GenBank: ABS17096.1) and Serratia proteamaculans (NCBI Reference Sequence: WP_174354643.1), as well as the gene encoding cysteine sulfinic acid decarboxylase from Tribolium castaneum (NCBI Reference Sequence: XM_966741.3) were codon optimized, synthesized and subcloned into pMPICZHis between the BstBI and NotI sites. The genes encoding cytosolic and mitochondrial aspartate aminotransferases (AAT) were PCR amplified from genomic DNA of P. pastoris GS115 using primers pair AAT1-BstBI(gb)-F/AAT1-NotI(gb)-R and AAT2-BstBI(gb)-F/AAT2-NotI(gb)-R, which was then cloned into pMPICKmHis between the BstBI and NotI sites using Gibson assembly method. Similarly, the $p p c$ and pck genes from E. coli BW25113 (GenBank: CP072663.1), as well as the pck gene from Actinobacillus succinogenes (GenBank: AY308832.1) were PCR amplified via the primers pair PPC-BstBI(gb)-F/PPCNotI(gb)-R, PCK-BstBI(gb)-F/PCK-NotI(gb)-R and AsPCK-BstBI(gb)-F/AsPCK-NotI(gb)-R and cloned into pMPIC2H between the BstBI and Not I sites.

A previously described multi-copy plasmid construction method (Yu et al. 2020a) was applied for the construction of the 2-, or 3-gene co-expression plasmids. For example, the pMPICZHis-ADC plasmid was doubledigested with $S p h \mathrm{I}$ and $\mathrm{BamHI}$ to generate the expression cassette 5'AOX1-ADC-3'AOX1, which was then inserted between the SphI and BglII sites of pMPICZHisADC to create pMPICZHis-2ADC. Similarly, the gene cassette 5'AOX1-Spe-3'AOX1 was reinserted between 
Table 2 Plasmids and strains constructed in this study

\begin{tabular}{|c|c|c|}
\hline Names & Relative characteristics & References \\
\hline \multicolumn{3}{|l|}{ Plasmids } \\
\hline pPICZA & Vector for extracellular expression recombinant protein carrying Zeo ${ }^{R}$ & Invitrogen \\
\hline $\mathrm{pAOa}$ & Vector for extracellular expression; derived from pPICZaA and pAO815, HIS4+ & Zhu et al. (2011) \\
\hline pMPICZHis & Extracellular expression vector carrying HIS4 gene, Zeo ${ }^{R}$ & This work \\
\hline pMPICKmHis & Replace antibiotic resistance marker (Zeocin) of pMPICZHis with kanamycin & This work \\
\hline $\mathrm{pMPIC} 2 \mathrm{H}$ & Replace antibiotic resistance marker (Zeocin) of pMPICZHis with hygromycin & This work \\
\hline pMPICZHis-bliADC & $\begin{array}{l}\text { Codon-optimized panD gene from Bacillus paralicheniformis ATCC 9945a cloned into pMPICZHis at BstBI and } \\
\text { Notl site }\end{array}$ & This work \\
\hline pMPICZHis-bsADC & Codon-optimized panD gene from Bacillus subtilis cloned into pMPICZHis at BstBI and Notl site & This work \\
\hline pMPICZHis-sgeADC & $\begin{array}{l}\text { Codon-optimized panD gene from Streptomyces griseorubiginosus cloned into pMPICZHis at BstBI and Notl } \\
\text { site }\end{array}$ & This work \\
\hline pMPICZHis-cguADC & Codon-optimized panD gene from Corynebacterium glutamicum cloned into pMPICZHis at BstBI and Notl site & This work \\
\hline pMPICZHis-TcCSADC & $\begin{array}{l}\text { Cysteine sulfinic acid decarboxylase from Tribolium castaneum (TCCSADC) cloned into pMPICZHis at BstBI and } \\
\text { Notl site }\end{array}$ & This work \\
\hline pMPICZHis-Oan & aspDH gene from Ochrobactrum anthropi ATCC 4918 cloned into pMPICZHis at BstBI and Notl site & This work \\
\hline pMPICZHis-Spe & aspDH gene from Serratia proteamaculans cloned into pMPICZHis at BstBI and Notl site & This work \\
\hline pMPICZHis-ADC-Oan & $\begin{array}{l}\text { panD expression cassette (pMPICZHis-ADC digest by Sphl and BamHI) cloned into pMPICZHis-Oan at Sphl } \\
\text { and Bg/ll site }\end{array}$ & This work \\
\hline pMPICZHis-ADC-Spe & $\begin{array}{l}\text { panD expression cassette (pMPICZHis-ADC digest by Sphl and BamHI) cloned into pMPICZHis-Spe at Sphl and } \\
\text { Bg/ll site }\end{array}$ & This work \\
\hline pMPICKmHis-AAT1 & Cytosolic aspartate aminotransferase from P. pastoris GS115 cloned into pMPICZHis at BstBI and Notl site & This work \\
\hline pMPICKmHis-AAT2 & Mitochondrial aspartate aminotransferase from P. pastoris GS115 cloned into pMPICZHis at BstBI and Notl site & This work \\
\hline pMPICZHis-2ADC & Two copies of panD gene expression cassette cloned into pMPICZHis & This work \\
\hline pMPICZHis-2ADC-Spe & $\begin{array}{l}\text { Two copies of panD gene expression cassette and one copy of SpeaspDH gene expression cassette cloned } \\
\text { into pMPICZHis }\end{array}$ & This work \\
\hline PMPIC2H-PPC & ppc gene from Escherichia coli cloned into integration vector pMPIC2H at BstBI and Notl site & This work \\
\hline PMPIC2H-AsPCK & pck gene from Actinobacillus succinogenes cloned into integration vector pMPIC2H at BstBI and Notl site & This work \\
\hline PMPIC2H-PCK & pck gene from Escherichia coli cloned into integration vector pMPIC2H at BstBI and Notl site & This work \\
\hline \multicolumn{3}{|l|}{ Strains } \\
\hline E. coli DH5a & Commercial transformation host for cloning & Invitrogen \\
\hline P. pastoris GS115 & Commercial transformation host for cloning; HIS4-, Mut + & Invitrogen \\
\hline$\Delta \mathrm{ku} 70$ & GS115, $\triangle K U 70 ; H I S 4+$ & This work \\
\hline BliADC & $\triangle$ ku70 harboring pMPICZHis-bliADC & This work \\
\hline BsADC & $\triangle \mathrm{ku} 70$ harboring pMPICZHis-bsADC & This work \\
\hline SgeADC & $\triangle$ ku70 harboring pMPICZHis-sgeADC & This work \\
\hline CguADC & $\triangle$ ku70 harboring pMPICZHis-cguADC & This work \\
\hline TCCSADC & $\triangle$ ku70 harboring pMPICZHis-TcCSADC & This work \\
\hline ADC-Oan & $\triangle$ ku70 harboring pMPICZHis-ADC-Oan & This work \\
\hline ADC-Spe & $\triangle$ ku70 harboring pMPICZHis-ADC-Spe & This work \\
\hline ADC-AAT1 & $\triangle$ ku70 harboring pMPICZHis-bsADC and pMPICKmHis-AAT1 & This work \\
\hline ADC-AAT2 & $\triangle$ ku70 harboring pMPICZHis-bsADC and pMPICKmHis-AAT2 & This work \\
\hline $2 A D C$ & $\triangle$ ku70 harboring pMPICZHis-2ADC & This work \\
\hline 2ADC-Spe & $\triangle \mathrm{ku} 70$ harboring pMPICZHis-2ADC-Spe & This work \\
\hline PPC & $\triangle \mathrm{ku} 70$ harboring pMPICZHis-2ADC-Spe and pMPIC2H-PPC & This work \\
\hline AsPCk & $\triangle$ ku70 harboring pMPICZHis-2ADC-Spe and pMPIC2H-AsPCK & This work \\
\hline PCK & $\triangle$ ku70 harboring pMPICZHis-2ADC-Spe and pMPIC2H-PCK & This work \\
\hline
\end{tabular}


the SphI and BglII sites of pMPICZHis-2ADC to create pMPICZHis-2ADC-Spe.

\section{Construction of recombinant strains}

The KU70 gene of the wild-type P. pastoris strain GS115 was deleted using the Cre-LoxP method to improve the homologous recombination efficiency (Guo et al. 2021; Weninger et al. 2018). The expression vectors pMPICZHis-bliADC, pMPICZHis-bsADC, pMPICZHis-sgeADC, pMPICZHis-cguADC, pMPICZHis-TcCSADC, pMPICZHis-ADC-Oan, pMPICZHis-ADC-Spe, pMPICZHis-2ADC, and pMPICZHis-2ADC-Spe were linearized with $B s p E I$ and used to transform the $\Delta \mathrm{ku} 70$ strain by electroporation. The linearized vectors were integrated into the chromosome of $\Delta \mathrm{ku} 70$ strain at the HIS4 gene locus. After integration of the linearized vector, the mutant HIS4 gene was repaired, enabling the recombinant strains growing on MD plate. Then, 8-16 colonies were picked for PCR verification and 3 correct colonies were randomly selected for fermentation analysis. Positive transformants were named BliADC, BsADC, SgeADC, CguADC, TcCSADC, ADC-Oan, ADC-Spe, 2ADC and 2ADC-Spe, respectively. The expression vectors pMPICKmHis-AAT1 and pMPICKmHis-AAT2 were linearized using $B s p E I$ and used to transform the BsADC strain to generate ADC-AAT1 and ADC-AAT2, respectively. The expression vectors pMPIC2H-PPC, pMPIC2H-AsPCK, and pMPIC2H-PCK were linearized with $B s p$ EI, StuI and $S s p$ I, respectively, and used to transform the strain 2ADC-Spe, yielding the recombinant strains PPC, AsPCK and PCK, respectively.

\section{$\beta$-Alanine production in shake-flask fermentations}

The strains were precultured in $10 \mathrm{~mL}$ YPD medium for $48 \mathrm{~h}$. This seed culture was centrifuged and resuspended with $25 \mathrm{~mL}$ of BMMY medium $(20 \mathrm{~g} / \mathrm{L}$ peptone, $10 \mathrm{~g} / \mathrm{L}$ yeast extract, $13.4 \mathrm{~g} / \mathrm{L} \mathrm{YNB}, 0.4 \mathrm{mg} / \mathrm{L}$ biotin, $8.7 \mathrm{~g} / \mathrm{L}$ monobasic potassium phosphate, $\mathrm{pH} 6.0$ ) in 250 -mL baffled shake flasks with an initial $\mathrm{OD}_{600} \approx 3.0$. The fermentation was carried out at $30^{\circ} \mathrm{C}$ and $220 \mathrm{rpm}$ for 6 days. Recombinant gene expression was induced by adding $200 \mu \mathrm{L}$ of pure methanol to each flask, followed by feeding with $200 \mu \mathrm{L}$ of methanol at $12 \mathrm{~h}$ intervals.

\section{$\beta$-Alanine production by fed-batch fermentation in a 1-L fermenter}

The strains were first grown in $50 \mathrm{~mL}$ YPD for $36 \mathrm{~h}$ and transferred into 1-L stirred tank reactors (Infors, Switzerland) containing $0.8 \mathrm{~L}$ of BMGY (20 g/L peptone, $10 \mathrm{~g} / \mathrm{L}$ yeast extract, $13.4 \mathrm{~g} / \mathrm{L}$ YNB, $0.4 \mathrm{mg} / \mathrm{L}$ biotin, $8.7 \mathrm{~g} / \mathrm{L}$ monobasic potassium phosphate, $40 \mathrm{~g} / \mathrm{L}$ glycerol, $\mathrm{pH}$ 6.0) supplemented with $4.0 \mathrm{~mL}$ PTM 1 trace salts $(6 \mathrm{~g} / \mathrm{L}$ $\mathrm{CuSO}_{4} \cdot 5 \mathrm{H}_{2} \mathrm{O}, 0.09 \mathrm{~g} / \mathrm{L} \mathrm{KI}, 3 \mathrm{~g} / \mathrm{L} \mathrm{MnSO}_{4} \cdot \mathrm{H}_{2} \mathrm{O}, 0.02 \mathrm{~g} / \mathrm{L}$
$\mathrm{H}_{3} \mathrm{BO}_{3}, 0.2 \mathrm{~g} / \mathrm{L} \mathrm{MoNa} \mathrm{O}_{4} \cdot 2 \mathrm{H}_{2} \mathrm{O}, 0.5 \mathrm{~g} / \mathrm{L} \mathrm{CoCl}, 20 \mathrm{~g} / \mathrm{L}$ $\mathrm{ZnCl}_{2}, 65 \mathrm{~g} / \mathrm{L} \mathrm{FeSO}_{4} \cdot 7 \mathrm{H}_{2} \mathrm{O}, 0.2 \mathrm{~g} / \mathrm{L}$ biotin, $5.0 \mathrm{~mL} / \mathrm{L}$ $\mathrm{H}_{2} \mathrm{SO}_{4}$ ). The temperature was set to $30{ }^{\circ} \mathrm{C}$, the $\mathrm{pH}$ was controlled at 6.0 by adding $\mathrm{NH}_{3} \cdot \mathrm{H}_{2} \mathrm{O}(28 \%$, v/v), the dissolved oxygen concentration was kept above $20 \%$ of the atmospheric value by varying the air flow rate between 0.5 and $2 \mathrm{~L} / \mathrm{min}$. The entire cultivation started with a batch phase lasting for $20-24 \mathrm{~h}$. Heterologous gene expression was induced by the addition of $0.25 \%(\mathrm{v} / \mathrm{v})$ methanol, and the methanol concentration was maintained at $3 \mathrm{~g} / \mathrm{L}$ throughout the entire fermentation span using an automatic methanol control station (FC2002, East China University of Science and Technology, Shanghai, China).

\section{Analytical methods}

The cell growth was analyzed by measuring the optical density at $600 \mathrm{~nm}$. $\beta$-Alanine production was measured by high-performance liquid chromatography (HPLC) with a variable wavelength detector (VWD) set to $334 \mathrm{~nm}$ and an Agilent ZRABOX SB-C18 column $(4.6 \mathrm{~mm} \times 250 \mathrm{~mm}, 5 \mu \mathrm{m})$ after centrifugation of the fermentation samples and o-phthalaldehyde (OPA) derivatization (Pei et al. 2017). The mobile phase consisted of $35 \mathrm{mM}$ sodium acetate $(\mathrm{pH} 7.5)$ containing $30 \%$ methanol with a flow rate of $1 \mathrm{~mL} / \mathrm{min}$.

\section{Results}

Overexpression of $A D C s$ from different sources

for the production of $\beta$-alanine and tolerance of the $P$. pastoris chassis to $\beta$-alanine

Since aspartate is the direct precursor for $\beta$-alanine synthesis via decarboxylation by ADCs, and was reported to have one of the largest precursor pool sizes among all amino acids in P. pastoris (Carnicer et al. 2012), we hypothesized that overexpression of ADC would lead to the accumulation of $\beta$-alanine. Nevertheless, only trace amount of extracellular aspartate $(\sim 80 \mathrm{mg} / \mathrm{L})$ were detected for the wild-type $P$. pastoris (data not shown) probably because aspartate is an intermediate metabolite and proteinogenic amino acid and thus is not prone to accumulate. Four genes encoding ADC from B. paralicheniformis, B. subtilis, S. griseorubiginosus, C. glutamicum, as well as the gene encoding cysteine sulfinic acid decarboxylase from $T$. castaneum, which was reported to possess higher decarboxylation activity than ADCs, were evaluated in this work. The coding sequences were individually placed under the control of the strong $A O X 1$ promoters and integrated into the genome of $P$. pastoris. In order to increase the efficiency of homologous recombination (HR), the KU70 mutant strain $(\Delta \mathrm{ku} 70)$ which has impaired nonhomologous end joining (NHEJ) was used as a parent strain (Guo et al. 

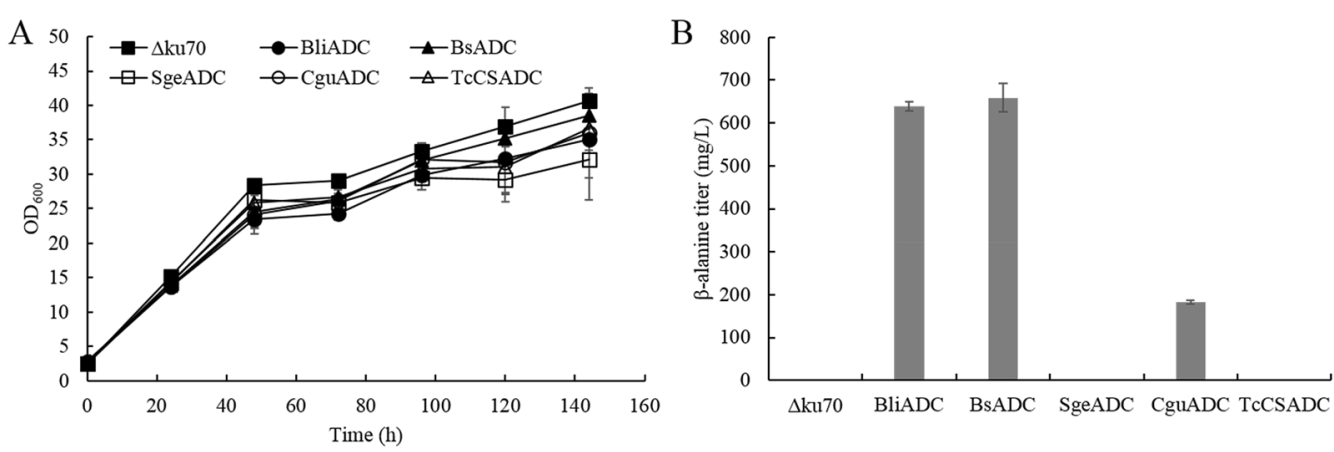

Fig. 2 Overexpression of ADCs from different sources for $\beta$-alanine synthesis. A Growth profiles of recombinant strains; $\mathbf{B} \beta$-alanine production in recombinant strains at the end of fermentation. Three parallel flasks are tested for each strain. Error bars represent deviations $(n=3)$

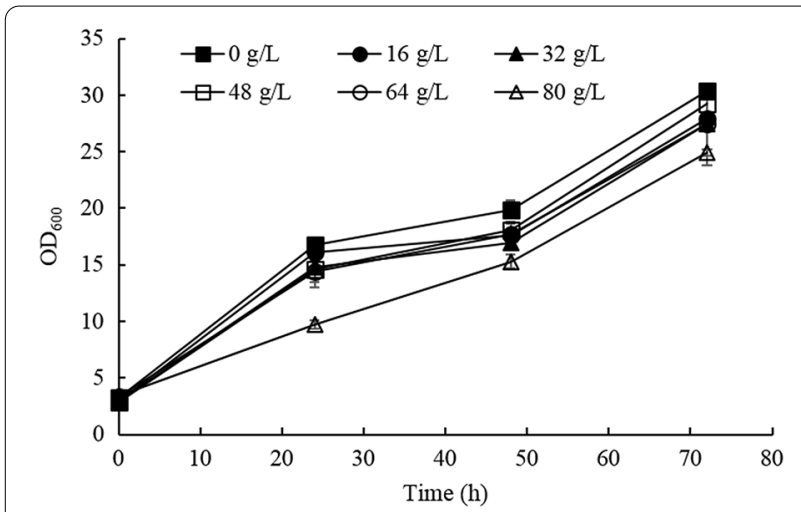

Fig. 3 Tolerance of wild-type P. pastoris strain for various concentrations of $\beta$-alanine

2021; Weninger et al. 2018). The individual overexpression of ADCs from B. paralicheniformis, B. subtilis and C. glutamicum enabled the accumulation of $\beta$-alanine after 6 days of fermentation when feeding the strains with methanol (Fig. 2B), indicating the functional protein expression of ADCs. The highest titer $(658.9 \mathrm{mg} / \mathrm{L})$ was reached by the recombinant strain expressing $B$. subtilis ADC (BsADC) (Fig. 2B), and BsADC was used as the starting strain for further metabolic engineering. To investigate whether the tolerance to $\beta$-alanine was a limiting factor for $\beta$-alanine production, $P$. pastoris was cultivated in media supplemented with different concentrations of $\beta$-alanine. The results showed that yeast cell growth was substantially reduced only in the presence of $80 \mathrm{~g} / \mathrm{L}$ of $\beta$-alanine $(P<0.01)$ at $72 \mathrm{~h}$, while the cell growth at other concentrations was not significantly different from the control $(P>0.05)$ (Fig. 3 ).

\section{Overexpression of AAT or AspDH to increase aspartate supply for improved $\beta$-alanine synthesis}

The conversion of oxaloacetate (OAA) into aspartate can be achieved by the transfer of an amino group from glutamate catalyzed by AAT, or direct amination with ammonium as the amino group donor catalyzed by AspDH. $P$. pastoris possesses a mitochondrial AAT (encoded by $A A T 1)$ and a cytosolic AAT (AAT2), and both genes were cloned into the pMPICKmHis vector and tested for their effects on $\beta$-alanine synthesis, respectively. In a previous paper, we reported that an AspDH from S. proteamaculans has high activity and stability (Li et al. 2017a). Thus, the SpeAspDH was also overexpressed in recombinant P. pastoris using the same strategy as AAT1 and AAT2. The shake-flask fermentation results showed that while neither AAT1 or AAT2 significantly improved $\beta$-alanine synthesis, the $\beta$-alanine titer was increased by $19.6 \%$ (to $787.9 \mathrm{mg} / \mathrm{L}$ ) by overexpressing SpeAspDH (Fig. 4B). In addition, no extracellular aspartate accumulation could be detected for all ADC overexpressed strains even after the optimization of the aspartate precursor supply (data not shown).

\section{Further improvement of $\beta$-alanine production by increasing the $A D C$ copy number}

After optimization of the aspartate precursor supply, we investigated whether aspartate decarboxylation is a potential bottleneck for $\beta$-alanine production by increasing the copy number of the encoding gene. Vectors harboring two copies of the ADC expression cassette alone and together with the SpeAspDH expression cassette were introduced into the $\Delta \mathrm{ku} 70$ strain, resulting in the recombinant strains $2 \mathrm{ADC}$ and $2 \mathrm{ADC}$-Spe, respectively. 

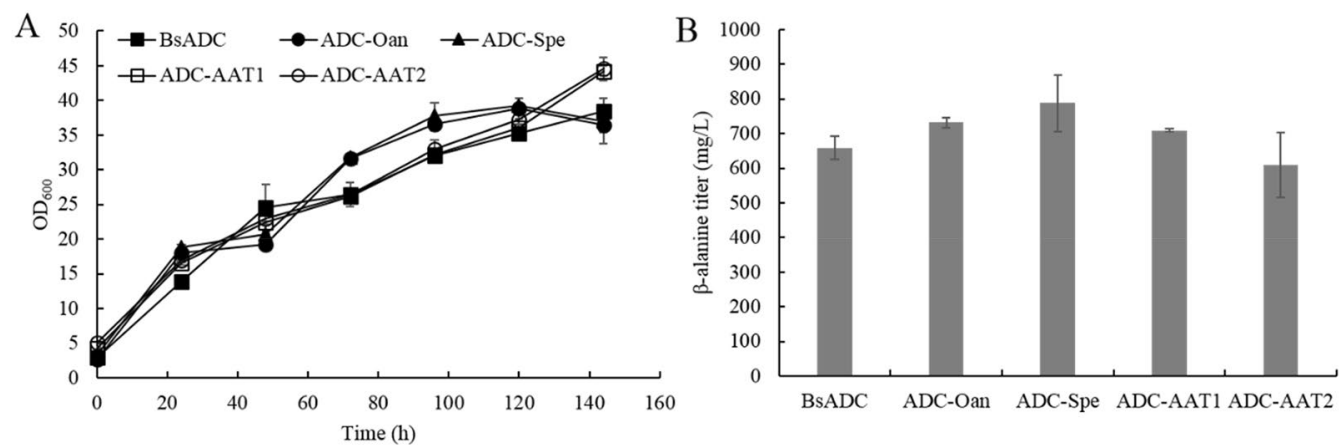

Fig. 4 The effects of overexpression of AAT or AspDH on $\beta$-alanine production. A Growth profiles of recombinant strains; $\mathbf{B} \beta$-alanine production in recombinant strains at the end of fermentation. Three parallel flasks are tested for each strain. Error bars represent deviations $(n=3)$
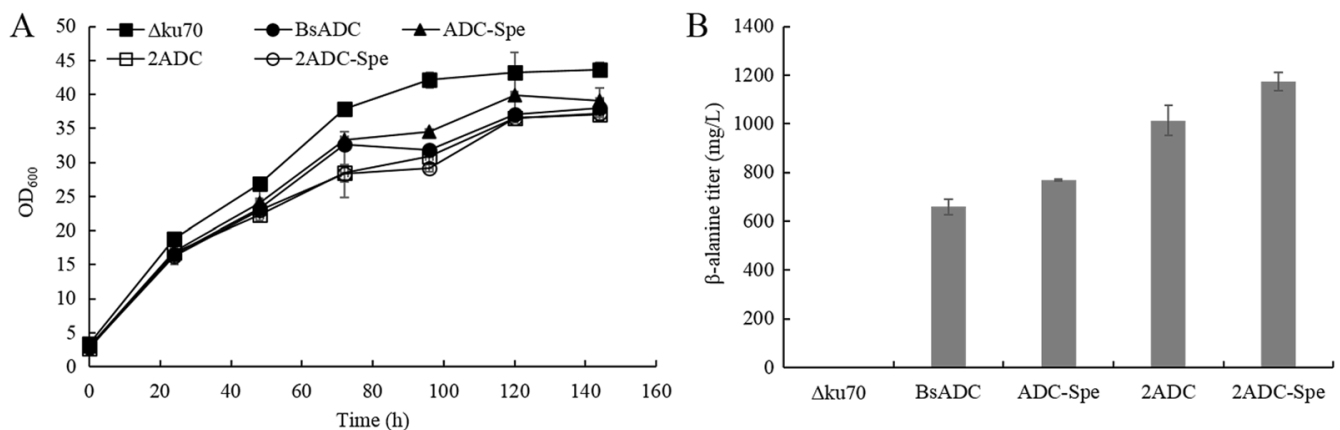

Fig. 5 The effects of increasing ADC copy number on $\beta$-alanine production. A Growth profiles of recombinant strains; $\mathbf{B} \beta$-alanine production in recombinant strains at the end of fermentation. Three parallel flasks are tested for each strain. Error bars represent deviations $(n=3)$

The multi-copy strains $2 \mathrm{ADC}$ and 2ADC-Spe exhibited 53.9 and $52.6 \%$ increases of $\beta$-alanine production compared with their respective single-copy counterparts, ADC and ADC-Spe (Fig. 5B). However, increase of ADC copy number to three did not lead to further increase of $\beta$-alanine (data not shown). Notably, the cell growth of all $\beta$-alanine producing strains (especially the two copy strains) were significantly slower than that of the wildtype strain (Fig. 5A), illustrating the negative effects of $\mathrm{ADC}$ overexpression on the physiology of yeast cells.

\section{Effect of strengthening phosphoenolpyruvate (PEP) carboxylation on $\beta$-alanine production}

A number of studies showed that $\mathrm{CO}_{2}$ fixation-based carboxylation of $\mathrm{C} 3$ metabolites plays an important role in the synthesis of $\mathrm{C} 4$ precursors (Tan et al. 2013). Therefore, the effect of strengthening PEP carboxylation on $\mathrm{C} 4$ precursor supply and thereby $\beta$-alanine synthesis was further investigated. Since PEP can be converted into OAA by either PEP carboxylase (PPC) or PEP carboxykinase (PCK), the encoding $p p c$ and $p c k$ genes from $E$. coli (Zhang et al. 2011), as well as $p c k$ gene (encoding the ATP forming PEP carboxykinase,
E.C: 4.1.1.49) from A. succinogenes (Hu et al. 2018), were individually overexpressed in 2ADC-Spe to evaluate their impact on $\beta$-alanine production in shake-flask fermentations. However, overexpression of $p p c$ did not lead to significant increase in the $\beta$-alanine titer compared to the control (2ADC-Spe), while both PCKs from $A$. succinogenes and $E$. coli decreased the $\beta$-alanine titer (by 26.4 and $27.6 \%$, respectively) (Fig. 6B).

\section{Fed-batch fermentation to improve $\beta$-alanine production} In order to obtain a higher $\beta$-alanine titer, fed-batch fermentation of the 2ADC-Spe strain was performed in a 1-L fermenter using a two-stage strategy. The fermentation was started with a glycerol phase $(40 \mathrm{~g} / \mathrm{L}$ glycerol) to facilitate biomass accumulation. When the glycerol was depleted and an $\mathrm{OD}_{600}$ of approximately 80 was reached, the methanol phase was induced by maintaining the methanol concentration at $3 \mathrm{~g} / \mathrm{L}$ using an on-line methanol analyzer. $\beta$-Alanine accumulation began in the methanol phase, and the highest $\beta$-alanine titer reached $5.6 \mathrm{~g} / \mathrm{L}$ (Fig. 7). However, the yeast cells grew very slowly during the entire methanol phase and 

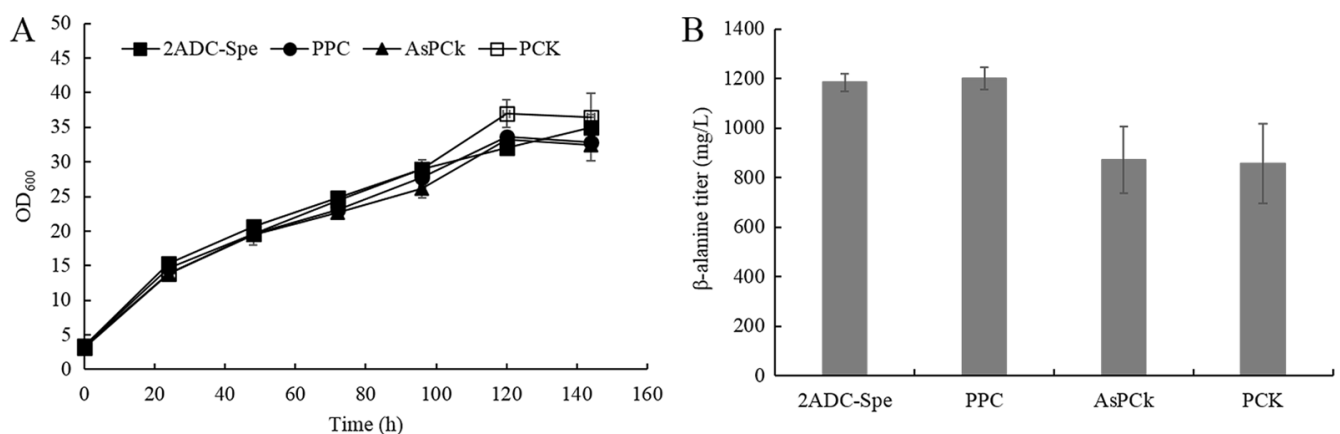

Fig. 6 The effects of strengthening PEP carboxylation on $\beta$-alanine production. A Growth profiles of recombinant strains; $\mathbf{B} \beta$-alanine production in recombinant strains at the end of fermentation. Three parallel flasks are tested for each strain. Error bars represent deviations $(n=3)$

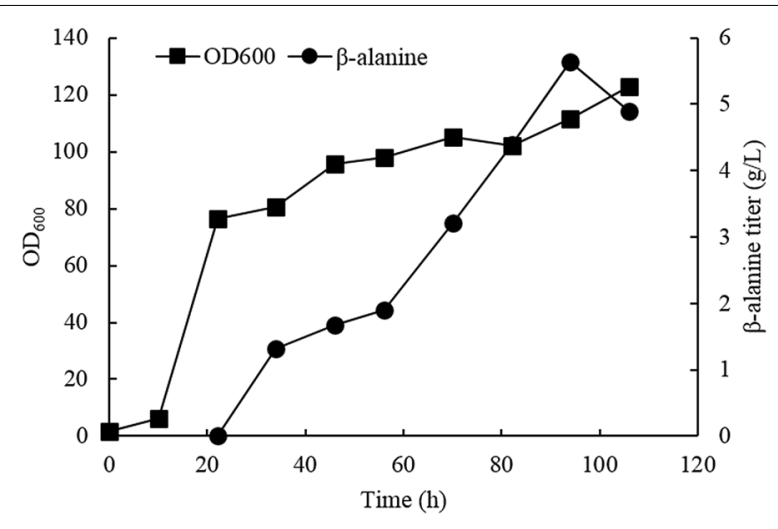

Fig. 7 Fed-batch fermentation profile of 2ADC-Spe strain

the biomass only reached an $\mathrm{OD}_{600}$ of 123 after $106 \mathrm{~h}$ (Fig. 7), corresponding to an average specific growth rate of $0.0044 \mathrm{~h}^{-1}$.

\section{Discussion}

Pichia pastoris is one of the most widely used eukaryotic expression systems for heterologous proteins, and in recent years, its potential as a cell factory for the production of chemicals is also receiving increasing attention (Zhu et al. 2019). As a native methylotroph, P. pastoris has unique advantages over $S$. cerevisiae and E. coli when methanol is used as a feedstock (Siripong et al. 2020; Siripong et al. 2018). Nevertheless, it is still challenging to engineer $P$. pastoris for the production of chemicals, largely because as a Crabtree-negative yeast, $P$. pastoris tends to accumulate biomass rather than produce metabolites. Reports on metabolite production in fermentations of engineered $P$. pastoris mainly remain at the proof-of-concept stage, with titers usually lower than $1 \mathrm{~g} / \mathrm{L}$, especially when methanol is used as substrate (Gao et al. 2021). Several recent studies reported gram per liter metabolites production in test tubes or shake flasks.
Yamada et al. (2019) reported a $3.48 \mathrm{~g} / \mathrm{L}$ of D-lactic acid production from $30 \mathrm{~g} / \mathrm{L}$ of methanol by engineered $P$. pastoris using YPM medium containing yeast extract and peptone. With an additional induction step by culturing engineered yeast cells in methanol complex medium (BMMY medium), Guo et al. (2021) achieved $0.75 \mathrm{~g} / \mathrm{L}$ and $2.79 \mathrm{~g} / \mathrm{L}$ of malic acid production in mineral-salt based fermentation medium with and without the addition of $1 \mathrm{~g} / \mathrm{L}$ of yeast extract, respectively. Unfortunately, the chemicals production potential of these recombinant $P$. pastoris strains was not fully evaluated by cultivation on the fermenter scale. In this study, we achieved a $\beta$-alanine titer of $1.2 \mathrm{~g} / \mathrm{L}$ using BMMY medium in shake-flask culture, which is the first demonstration of amino acids production from methanol with $P$. pastoris as the chassis. More importantly, the production of the strain was further evaluated in a 1-L fermenter using a two-stage strategy with a high initial biomass. Finally, $5.6 \mathrm{~g} / \mathrm{L}$ of $\beta$-alanine titer was obtained, which represents the highest metabolite production titer ever reached in $P$. pastoris using methanol as the substrate.

Aspartate decarboxylation is the most important step of $\beta$-alanine synthesis. Although ADCs from different sources have been evaluated for $\beta$-alanine synthesis in $E$. coli (Feng et al. 2019; Liu et al. 2019; Pei et al. 2017; Song et al. 2015), the protein expression levels and enzyme activities of these ADCs may be different in eukaryotic hosts such as $P$. pastoris. Accordingly, we evaluated bacterial ADCs from different sources, including ones known to function well in $E$. coli such as the ADCs from C. glutamicum (Song et al. 2015) and B. subtilis (Pei et al. 2017), as well as the recently reported insect TcCSADC, which is reported to be a dimer that is resistant to turnover-dependent inactivation observed in ADCs from prokaryotes (Liu et al. 2019; Yu et al. 2020b). Only ADCs from Bacillus spp. exhibited high activity in the P. pastoris system, and the mere expression of B. subtilis ADC 
resulted in an initial success in $\beta$-alanine accumulation. Moreover, we showed that $\beta$-alanine production can be remarkably improved by doubling the ADC copy number, indicating that even with the strong $A O X 1$ promoter, the decarboxylation of aspartate still remains the bottleneck for $\beta$-alanine synthesis. Increasing the copy number of the target gene is a widely used strategy in recombinant protein expression in P. pastoris (Yu et al. 2020a). Our work suggested that this strategy is still important in tuning metabolic pathways for chemical synthesis.

Increasing the aspartate supply is also crucial for achieving high $\beta$-alanine production. To this end, two strategies were applied. First, we aimed at increasing the conversion of OAA into aspartate by screening for appropriate enzymes for reductive amination. Although the overexpression of AAT was widely used to increase aspartate flux for the production of aspartate family amino acids (AFAAs) in industrial microbes such as C. glutamicum and E. coli (Li et al. 2017a, b; Piao et al. 2019), the efficacy of AspDH overexpression for enhancing AFAAs production was rarely. The present study demonstrates that the overexpression of SpeAspDH can significantly increase $\beta$-alanine production, corroborating the potential of AspDHs for the production of AFAAs. The second strategy is to increase OAA supply by strengthening $\mathrm{C} 3$ carboxylation. PPC exhibits high affinity for bicarbonate and high catalytic velocity in the carboxylation of PEP, but the energy contained in PEP is lost in this reaction with the release of inorganic phosphate. Conversely, PCK can conserve the high energy of PEP, leading to net production of ATP for growth and cell maintenance, but it has low affinity for bicarbonate and relatively low catalytic velocity (Tan et al. 2013). In this study, the overexpression of PPC from $E$. coli only led to a slight increase of the $\beta$-alanine titer, and $p c k$ overexpression even decreased $\beta$-alanine production, suggesting that OAA supply may not be the bottleneck for $\beta$-alanine synthesis at the current stage, and there might be other rate-limiting factors that should be resolved in future studies.

Another noteworthy phenomenon observed in this work is that the growth of the cells was remarkably decreased, especially for strains harboring two copies of ADC. After shifting to the methanol phase in the fermenter, the yeast cells grew very slowly, with an average specific growth rate of $0.0044 \mathrm{~h}^{-1}$, which was an order of magnitude lower than that of the wildtype strain under the same conditions (usually more than $0.04 \mathrm{~h}^{-1}$ ). The impaired cell growth of the ADCexpressing strain is less likely caused by product inhibition, because the $\beta$-alanine titer far below its inhibitory concentration. Nevertheless, this phenomenon is not quite unexpected because overexpression of ADC may cause considerable metabolic burden on $P$. pastoris. Moreover, as an important metabolic intermediate, aspartate takes part in many biological processes, such as the synthesis of AFAAs ( $\mathrm{Li}$ et al. 2017b; Park and Lee 2010), protecting microbes against acid stress (Wu et al. 2013), shuttling of redox equivalents (Bakker et al. 2001 ), etc., and depletion of aspartate for $\beta$-alanine synthesis may cause severe perturbations of the normal physiology of yeast cells. The underlying mechanism is currently under investigation using omics approaches.

\section{Conclusions}

Recombinant $P$. pastoris strains were constructed for the production of $\beta$-alanine from methanol by screening and overexpressing ADCs from different sources. The $\beta$-alanine titer was further increased by increasing ADC copy number and overexpression of AspDH. The production potential of the best strain 2ADC-Spe was evaluated on $1-\mathrm{L}$ fermenter and $5.6 \mathrm{~g} / \mathrm{L}$ of $\beta$-alanine titer was obtained, which is the highest metabolite production titer ever reached in $P$. pastoris using methanol as the substrate. This work is the first attempt to produce amino acids from methanol using recombinant $P$. pastoris as the cell chassis.

\section{Abbreviations \\ ADC: Aspartate-a-decarboxylases; AspA: Aspartate ammonia-lyase; AAT: Aspar- tate aminotransferase; AspDH: Aspartate dehydrogenase; HR: Homologous recombination; NHEJ: Nonhomologous end joining; OAA: Oxaloacetate; PEP: Phosphoenolpyruvate; PPC: PEP carboxylase; PCK: PEP carboxykinase; AFAAs: Aspartate family amino acids; AOX: Alcohol oxidase.}

\section{Acknowledgements}

Not applicable.

\section{Authors' contributions}

TCZ and YL conceived the study and revised the manuscript. LTM designed and carried out the experiments, and drafted the manuscript. All authors read and approved the final manuscript.

\section{Funding}

This work was supported by the National Key R\&D Program of China (2018YFA0901400), Tianjin Synthetic Biotechnology Innovation Capacity Improvement Project (TSBICIP-KJGG-006-19) and the National Natural Science Foundation of China (No. 31970039).

Availability of data and materials

All data generated or analyzed during this study are included in this article.

\section{Declarations}

Ethics approval and consent to participate Not applicable.

\section{Consent for publication}

All authors approved the consent for publishing the manuscript to Bioresources and Bioprocessing. 


\section{Competing interests}

The authors declare that they have no competing interests.

\section{Author details}

${ }^{1}$ CAS Key Laboratory of Microbial Physiological and Metabolic Engineering, State Key Laboratory of Microbial Resources, Institute of Microbiology, Chinese Academy of Sciences, Beijing 100101, People's Republic of China. ${ }^{2}$ University of Chinese Academy of Sciences, Beijing 100049, People's Republic of China.

Received: 23 June 2021 Accepted: 8 September 2021

Published online: 22 September 2021

\section{References}

Bakker BM, Overkamp KM, van Maris AJ, Kötter P, Luttik MA, van Dijken JP Pronk JT (2001) Stoichiometry and compartmentation of NADH metabolism in Saccharomyces cerevisiae. FEMS Microbiol Rev 25(1):15-37. https:// doi.org/10.1111/j.1574-6976.2001.tb00570.x

Carlson GH (1943) Preparation of beta-alanine. US Patents 2,336,067, 7 Dec. 1943

Carnicer M, Canelas AB, Ten Pierick A, Zeng Z, van Dam J, Albiol J, Ferrer P, Heijnen JJ, van Gulik W (2012) Development of quantitative metabolomics for Pichia pastoris. Metabolomics 8(2):284-298. https://doi.org/10.1007/ s11306-011-0308-1

Feng Z, Zhang J, Chen G, Ge Y, Zhang X, Zhu H (2019) Extracellular expression of L-aspartate-alpha-decarboxylase from Bacillus tequilensis and its application in the biosynthesis of beta-alanine. Appl Biochem Biotechnol 189(1):273-283. https://doi.org/10.1007/s12010-019-03013-1

Ford JH (1945) The alkaline hydrolysis of $\beta$-aminopropionitrile. J Am Chem Soc 67(5):876-877. https://doi.org/10.1021/ja01221a503

Gao J, Jiang L, Lian J (2021) Development of synthetic biology tools to engineer Pichia pastoris as a chassis for the production of natural products. Synth Syst Biotechnol 6(2):110-119. https://doi.org/10.1016/j.synbio. 2021.04.005

Guo F, Dai Z, Peng W, Zhang S, Zhou J, Ma J, Dong W, Xin F, Zhang W, Jiang M (2021) Metabolic engineering of Pichia pastoris for malic acid production from methanol. Biotechnol Bioeng 118(1):357-371. https://doi.org/10. 1002/bit.27575

Harris RC, Tallon MJ, Dunnett M, Boobis L, Coakley J, Kim HJ, Fallowfield JL, Hill CA, Sale C, Wise JA (2006) The absorption of orally supplied beta-alanine and its effect on muscle carnosine synthesis in human vastus lateralis. Amino Acids 30(3):279-289. https://doi.org/10.1007/s00726-006-0299-9

Hu G, Zhou J, Chen X, Qian Y, Gao C, Guo L, Xu P, Chen W, Chen J, Li Y, Liu L (2018) Engineering synergetic CO2-fixing pathways for malate production. Metab Eng 47:496-504. https://doi.org/10.1016/j.ymben.2018.05. 007

Jin X, Zhang W, Wang Y, Sheng J, Xu R, Li J, Du G, Kang Z (2021) Biosynthesis of non-animal chondroitin sulfate from methanol using genetically engineered Pichia pastoris. Green Chem. https://doi.org/10.1039/d1 gc00260k

Li H, Lu X, Chen K, Yang J, Zhang A, Wang X, Ouyang P (2018) $\beta$-alanine production using whole-cell biocatalysts in recombinant Escherichia coli. Molecular Catalysis 449:93-98. https://doi.org/10.1016/j.mcat.2018.02.008

Li H, Zhu T, Miao L, Zhang D, Li Y, Li Q, Li Y (2017a) Discovery of novel highly active and stable aspartate dehydrogenases. Sci Rep 7(1):7881. https:// doi.org/10.1038/s41598-017-05522-7

LiY, Wei H, Wang T, Xu Q, Zhang C, Fan X, Ma O, Chen N, Xie X (2017b) Current status on metabolic engineering for the production of I-aspartate family amino acids and derivatives. Bioresour Technol 245(Pt B):1588-1602. https://doi.org/10.1016/j.biortech.2017.05.145

Liu Y, Cruz-Morales P, Zargar A, Belcher MS, Pang B, Englund E, Dan Q, Yin K, Keasling JD (2021) Biofuels for a sustainable future. Cell 184(6):1636-1647. https://doi.org/10.1016/j.cell.2021.01.052

Liu Z, Zheng W, Ye W, Wang C, Gao Y, Cui W, Zhou Z (2019) Characterization of cysteine sulfinic acid decarboxylase from Tribolium castaneum and its application in the production of beta-alanine. Appl Microbiol Biotechnol 103(23-24):9443-9453. https://doi.org/10.1007/s00253-019-10139-z

Ohara T, Sato T, Shimizu N, Prescher G, Schwind H, Weiberg O, Marten K, Greim H (2011) Acrylic acid and derivatives. In: Ullmann's Encyclopedia of Industrial Chemistry. https://doi.org/10.1002/14356007.a01_161.pub4
Park JH, Lee SY (2010) Metabolic pathways and fermentative production of L-aspartate family amino acids. Biotechnol J 5(6):560-577. https://doi.org/ 10.1002/biot.201000032

Pei W, Zhang J, Deng S, Tigu F, Li Y, Li Q, Cai Z, Li Y (2017) Molecular engineering of L-aspartate-alpha-decarboxylase for improved activity and catalytic stability. Appl Microbiol Biotechnol 101(15):6015-6021. https://doi.org/ 10.1007/s00253-017-8337-y

Piao X, Wang L, Lin B, Chen H, Liu W, Tao Y (2019) Metabolic engineering of Escherichia coli for production of L-aspartate and its derivative betaalanine with high stoichiometric yield. Metab Eng 54:244-254. https:// doi.org/10.1016/j.ymben.2019.04.012

Qian Y, Liu J, Song W, Chen X, Luo Q, Liu L (2018) Production of $\beta$-alanine from fumaric acid using a dual-enzyme cascade. ChemCatChem 10(21):49844991. https://doi.org/10.1002/cctc.201801050

Sale C, Saunders B, Harris RC (2010) Effect of beta-alanine supplementation on muscle carnosine concentrations and exercise performance. Amino Acids 39(2):321-333. https://doi.org/10.1007/s00726-009-0443-4

Shen Y, Zhao L, Li Y, Zhang L, Shi G (2014) Synthesis of $\beta$-alanine from I-aspartate using l-aspartate-a-decarboxylase from Corynebacterium glutamicum. Biotech Lett 36(8):1681-1686. https://doi.org/10.1007/ s10529-014-1527-0

Siripong W, Angela C, Tanapongpipat S, Runguphan W (2020) Metabolic engineering of Pichia pastoris for production of isopentanol (3-Methyl1-butanol). Enzyme Microb Technol 138:109557. https://doi.org/10. 1016/j.enzmictec.2020.109557

Siripong W, Wolf P, Kusumoputri TP, Downes JJ, Kocharin K, Tanapongpipat S, Runguphan W (2018) Metabolic engineering of Pichia pastoris for production of isobutanol and isobutyl acetate. Biotechnol Biofuels. https://doi. org/10.1186/s13068-017-1003-x

Song CW, Lee J, Ko YS, Lee SY (2015) Metabolic engineering of Escherichia coli for the production of 3-aminopropionic acid. Metab Eng 30:121-129. https://doi.org/10.1016/j.ymben.2015.05.005

Steunenberg P, Könst PM, Scott EL, Franssen MCR, Zuilhof H, Sanders JPM (2013) Polymerisation of $\beta$-alanine through catalytic ester-amide exchange. Eur Polymer J 49(7):1773-1781. https://doi.org/10.1016/j.eurpo lymj.2013.03.032

Tan Z, Zhu X, Chen J, Li Q, Zhang X (2013) Activating phosphoenolpyruvate carboxylase and phosphoenolpyruvate carboxykinase in combination for improvement of succinate production. Appl Environ Microbiol 79(16):4838-4844. https://doi.org/10.1128/AEM.00826-13

Tigu F, Zhang J, Liu G, Cai Z, Li Y (2018) A highly active pantothenate synthetase from Corynebacterium glutamicum enables the production of D-pantothenic acid with high productivity. Appl Microbiol Biotechnol 102(14):6039-6046. https://doi.org/10.1007/s00253-018-9017-2

Tomita H, Yokooji Y, Ishibashi T, Imanaka T, Atomi H (2014) An archaeal glutamate decarboxylase homolog functions as an aspartate decarboxylase and is involved in $\beta$-alanine and coenzyme A biosynthesis. J Bacteriol 196(6):1222-1230. https://doi.org/10.1128/jb.01327-13

Tuyishime P, Wang Y, Fan L, Zhang Q, Li Q, Zheng P, Sun J, Ma Y (2018) Engineering Corynebacterium glutamicum for methanol-dependent growth and glutamate production. Metab Eng 49:220-231. https://doi.org/10. 1016/j.ymben.2018.07.011

Wang L, Piao X, Cui S, Hu M, Tao Y (2020) Enhanced production of beta-alanine through co-expressing two different subtypes of L-aspartate-alphadecarboxylase. J Ind Microbiol Biotechnol 47(6-7):465-474. https://doi. org/10.1007/s10295-020-02285-5

Weninger A, Fischer JE, Raschmanova H, Kniely C, Vogl T, Glieder A (2018) Expanding the CRISPR/Cas9 toolkit for Pichia pastoris with efficient donor integration and alternative resistance markers. J Cell Biochem 119(4):3183-3198. https://doi.org/10.1002/jcb.26474

Whitaker WB, Jones JA, Bennett RK, Gonzalez JE, Vernacchio VR, Collins SM, Palmer MA, Schmidt S, Antoniewicz MR, Koffas MA, Papoutsakis ET (2017) Engineering the biological conversion of methanol to specialty chemicals in Escherichia coli. Metab Eng 39:49-59. https://doi.org/10.1016/j.ymben. 2016.10.015

Wu C, Zhang J, Du G, Chen J (2013) Aspartate protects Lactobacillus casei against acid stress. Appl Microbiol Biotechnol 97(9):4083-4093. https:// doi.org/10.1007/s00253-012-4647-2

Yamada R, Ogura K, Kimoto Y, Ogino H (2019) Toward the construction of a technology platform for chemicals production from methanol: $d$-lactic 
acid production from methanol by an engineered yeast Pichia pastoris. World J Microbiol Biotechnol. https://doi.org/10.1007/s11274-019-2610-4

Yu S, Miao L, Huang H, Li Y, Zhu T (2020a) High-level production of glucose oxidase in Pichia pastoris: Effects of Hac1 p overexpression on cell physiology and enzyme expression. Enzyme Microb Technol 141:109671. https://doi. org/10.1016/j.enzmictec.2020.109671

Yu XJ, Huang CY, XU XD, Chen H, Liang MJ, Xu ZX, Xu HX, Wang Z (2020b) Protein engineering of a pyridoxal-5'-phosphate-dependent l-aspartatealpha-decarboxylase from Tribolium castaneum for beta-alanine production. Molecules. https://doi.org/10.3390/molecules25061280

Zhang X, Wang X, Shanmugam KT, Ingram LO (2011) L-malate production by metabolically engineered Escherichia coli. Appl Environ Microbiol 77(2):427-434. https://doi.org/10.1128/AEM.01971-10

Zhu T, You L, Gong F, Xie M, Xue Y, Li Y, Ma Y (2011) Combinatorial strategy of sorbitol feeding and low-temperature induction leads to high-level production of alkaline beta-mannanase in Pichia pastoris. Enzyme Microb Technol 49(4):407-412. https://doi.org/10.1016/j.enzmictec.2011.06.022

Zhu T, Sun H, Wang M, Li Y (2019) Pichia pastoris as a versatile cell factory for the production of industrial enzymes and chemicals: Current status and future perspectives. Biotechnol J 14(6):e1800694. https://doi.org/10.1002/ biot.201800694
Zhu T, Zhao T, Bankefa OE, LiY (2020) Engineering unnatural methylotrophic cell factories for methanol-based biomanufacturing: Challenges and opportunities. Biotechnol Adv 39:107467. https://doi.org/10.1016/j.biote chadv.2019.107467

Zhu WL, Cui JY, Cui LY, Liang WF, Yang S, Zhang C, Xing XH (2016) Bioconversion of methanol to value-added mevalonate by engineered Methylobacterium extorquens AM1 containing an optimized mevalonate pathway. Appl Microbiol Biotechnol 100(5):2171-2182. https://doi.org/10.1007/ s00253-015-7078-z

Zou X, Guo L, Huang L, Li M, Zhang S, Yang A, Zhang Y, Zhu L, Zhang H, Zhang J, Feng Z (2020) Pathway construction and metabolic engineering for fermentative production of beta-alanine in Escherichia coli. Appl Microbiol Biotechnol 104(6):2545-2559. https://doi.org/10.1007/ s00253-020-10359-8

\section{Publisher's Note}

Springer Nature remains neutral with regard to jurisdictional claims in published maps and institutional affiliations.

\section{Submit your manuscript to a SpringerOpen ${ }^{\odot}$ journal and benefit from:}

- Convenient online submission

- Rigorous peer review

- Open access: articles freely available online

- High visibility within the field

- Retaining the copyright to your article

Submit your next manuscript at $\gg$ springeropen.com 